\title{
A MEAN VALUE THEOREM FOR $N$-SIMPLE FUNCTIONALS IN THE SENSE OF POPOVICIU
}

\section{IOAN GAVREA, MIRCEA IVAN, AND VICUTA NEAGOS}

Received 30 December, 2016

\begin{abstract}
We establish some mean value theorems involving $n$-simple functionals in the sense of Popoviciu. In particular, we obtain the Kowalewski mean value formula.

2010 Mathematics Subject Classification: 26A24

Keywords: mean value theorems, divided differences, finite differences
\end{abstract}

\section{PRELIMINARIES AND AUXILIARY RESULTS}

Throughout the paper $n \geq 1$ denotes an integer. Let $[a, b]$ be a real interval and $a \leq x_{0}<\cdots<x_{n} \leq b$.

\subsection{The divided difference}

In most books on numerical analysis, the divided differences of a function $f:\left\{x_{0}, \ldots, x_{n}\right\} \rightarrow \mathbb{R}$ are defined recursively:

$$
\begin{gathered}
{\left[x_{i} ; f\right]:=f\left(x_{i}\right), \quad i=0, \ldots, n,} \\
{\left[x_{0}, \ldots, x_{k} ; f\right]=\frac{\left[x_{1}, \ldots, x_{k} ; f\right]-\left[x_{0}, \ldots, x_{k-1} ; f\right]}{x_{k}-x_{0}}, \quad k=1, \ldots, n,}
\end{gathered}
$$

but can be written also in terms of determinants,

$$
\left[x_{0}, \ldots, x_{n} ; f\right]=\frac{1}{V\left(x_{0}, \ldots, x_{n}\right)}\left|\begin{array}{ccccc}
1 & x_{0} & \cdots & x_{0}^{n-1} & f\left(x_{0}\right) \\
1 & x_{1} & \cdots & x_{1}^{n-1} & f\left(x_{1}\right) \\
\vdots & \vdots & \ddots & \vdots & \vdots \\
1 & x_{n} & \cdots & x_{n}^{n-1} & f\left(x_{n}\right)
\end{array}\right|,
$$

where $V\left(x_{0}, \ldots, x_{n}\right)$ denotes the Vandermonde determinant

$$
\left|\begin{array}{cccc}
1 & x_{0} & \cdots & x_{0}^{n} \\
1 & x_{1} & \cdots & x_{1}^{n} \\
\vdots & \vdots & \ddots & \vdots \\
1 & x_{n} & \cdots & x_{n}^{n}
\end{array}\right|
$$




\subsection{Convexity of high order}

The standard definition of $n$-convex functions is that through divided differences (see, e.g., [6]). A function $f:[a, b] \rightarrow \mathbb{R}$ is said to be $n$-convex if

$$
\left[x_{0}, \ldots, x_{n} ; f\right]>0 \text {, }
$$

for any pairwise distinct points $x_{0}, \ldots, x_{n} \in[a, b]$.

\subsection{Simple form functionals}

Let $e_{i}:[a, b] \rightarrow \mathbb{R}, e_{i}(x)=x^{i}, i=0,1, \ldots, n$. The definition and the theorem rewritten below belong to Popoviciu.

Definition $1([7,9])$. A linear functional $A: C[a, b] \rightarrow \mathbb{R}$ is said to be $n$-simple in the sense of Popoviciu if:

(i) $A\left(e_{0}\right)=A\left(e_{1}\right)=\cdots=A\left(e_{n-1}\right)=0$,

(ii) $A(f) \neq 0$ for any $n$-convex function $f \in C[a, b]$.

In particular, $A\left(e_{n}\right) \neq 0$.

A connection between divided differences and $n$-simple functionals is supplied by the following celebrated result of Popoviciu.

Theorem 1 ([7,9, Popoviciu]). A linear functional $A: C[a, b] \rightarrow \mathbb{R}$ is $n$-simple if and only if for any $f \in C[a, b]$, there exist distinct points $\xi_{0}, \ldots, \xi_{n} \in(a, b)$ such that

$$
A(f)=A\left(e_{n}\right)\left[\xi_{0}, \ldots, \xi_{n} ; f\right] .
$$

\subsection{The finite difference operator}

The finite difference with step $h(0<h \leq(b-a) / n)$ of the function $f:[a, b] \rightarrow \mathbb{R}$ at the point $x \in[a, b)$ is defined by

$$
\Delta_{h}^{n} f(x)=\sum_{k=0}^{n}(-1)^{k}\left(\begin{array}{l}
n \\
k
\end{array}\right) f(x+(n-k) h) .
$$

Recall that finite difference can be written as a divided difference on equidistant points:

$$
\Delta_{h}^{n} f(x)=n ! h^{n}[x, x+h, \ldots, x+n h ; f] .
$$

Let $f$ possess a derivative of order $n$ on the interval $(a, b)$ and $x, x+n h \in[a, b]$.

By using the Lagrange mean value theorem, one can prove, step by step, that there exist $c_{k} \in(x, x+n h), k=1,2, \ldots, n$, such that:

$$
\Delta_{h}^{n} f(x)=h \Delta_{h}^{n-1} f^{\prime}\left(c_{1}\right)=\cdots=h^{k} \Delta_{h}^{n-k} f^{(k)}\left(c_{k}\right)=\cdots=h^{n} \Delta_{h}^{0} f^{(n)}\left(c_{n}\right),
$$

where $\Delta_{h}^{0} f^{(n)}\left(c_{n}\right)=f^{(n)}\left(c_{n}\right)$

One connection between the divided difference and the $n$-th derivative of a function is given by a classical result of Cauchy (see, e.g., [11, Theorem 2.10]): 
Proposition 1 (Cauchy). Let $f \in C^{n}[a, b]$ and $x_{0}, \ldots, x_{n}$ be pairwise distinct points in $[a, b]$. Then there exists at least one point $\xi \in[a, b]$ such that

$$
\left[x_{0}, \ldots, x_{n} ; f\right]=\frac{f^{(n)}(\xi)}{n !} .
$$

A much deeper mean value property for divided differences is provided by the following theorem of Popoviciu.

Proposition 2 ([8, Popoviciu (1954)]). If the function $f$ is continuous on an interval $[a, b]$ containing the distinct points $x_{0}, \ldots, x_{n}$, then there exists $c \in(a, b)$ and $h>0$ such that

$$
\left[x_{0}, \ldots, x_{n} ; f\right]=\frac{\Delta_{h}^{n} f(c)}{n ! h^{n}} .
$$

The following result is a generalization of Cauchy's mean-value theorem:

Theorem 2 ([3, Kowalewski (1932), p. 16] and [10, Raikov (1939)]). If

$f, g:[a, b] \rightarrow \mathbb{R}$ are continuous and $n$ times differentiable on $(a ; b)$, and $g^{(n)}(x) \neq 0$ for any $x \in(a, b)$, then there exists a point $c \in(a, b)$ such that

$$
\frac{\left[x_{0}, \ldots, x_{n} ; f\right]}{\left[x_{0}, \ldots, x_{n} ; g\right]}=\frac{f^{(n)}(c)}{g^{(n)}(c)} .
$$

For the purpose of simplicity, and without loss of generality, we will drop out the condition $g^{(n)}(x) \neq 0$ for any $x \in(a, b)$ and consider the following form of the previous equation,

$$
\left[x_{0}, \ldots, x_{n} ; f\right] g^{(n)}(c)=\left[x_{0}, \ldots, x_{n} ; g\right] f^{(n)}(c) .
$$

\section{MAIN RESULTS}

We give a simple elementary self-contained proof of Kowalewski's mean value formula (1.4) (see also [4]). Furthermore, we generalize (1.4) in two directions. Firstly, the divided differences are replaced with an $n$-simple functional. Secondly, we take a combination of forward differences and derivatives in place of the $n$-th derivatives in (1.4).

Consider the auxiliary function $H:[a, b] \rightarrow \mathbb{R}$,

$$
H(x)=\left|\begin{array}{cccccc}
1 & x_{0} & \cdots & x_{0}^{n-1} & f\left(x_{0}\right) & g\left(x_{0}\right) \\
1 & x_{1} & \cdots & x_{1}^{n-1} & f\left(x_{1}\right) & g\left(x_{1}\right) \\
\vdots & \vdots & \ddots & \vdots & \vdots & \vdots \\
1 & x_{n} & \cdots & x_{n}^{n-1} & f\left(x_{n}\right) & g\left(x_{n}\right) \\
1 & x & \cdots & x^{n-1} & f(x) & g(x)
\end{array}\right| .
$$


It is obvious that $H\left(x_{i}\right)=0, i=0,1, \ldots, n$. By using the Generalized Rolle Theorem, we deduce that there exists a point $c \in\left(x_{0}, x_{n}\right)$ such that $H^{(n)}(c)=0$, i.e.,

$$
0=\left|\begin{array}{cccccc}
1 & x_{0} & \cdots & x_{0}^{n-1} & f\left(x_{0}\right) & g\left(x_{0}\right) \\
1 & x_{1} & \cdots & x_{1}^{n-1} & f\left(x_{1}\right) & g\left(x_{1}\right) \\
\vdots & \vdots & \ddots & \vdots & \vdots & \vdots \\
1 & x_{n} & \cdots & x_{n}^{n-1} & f\left(x_{n}\right) & g\left(x_{n}\right) \\
0 & 0 & \cdots & 0 & f^{(n)}(c) & g^{(n)}(c)
\end{array}\right| .
$$

By expanding the previous determinant in terms of the last row, and dividing the result by the Vandermonde determinant $V\left(x_{0}, \ldots, x_{n}\right)$, the proof is complete.

The following result is a generalization of Kowalewski's mean value formula (1.4).

Theorem 3. Let $A: C[a, b] \rightarrow \mathbb{R}$ be an $n$-simple functional. and suppose that $f, g:[a, b] \rightarrow \mathbb{R}$ are continuous and possess derivatives of suitable order on $(a, b)$. Then there exist points $c_{k} \in(a, b), k=0, \ldots, n$, such that

$$
A(f) \Delta_{h}^{n-k} g^{(k)}\left(c_{k}\right)=A(g) \Delta_{h}^{n-k} f^{(k)}\left(c_{k}\right) .
$$

In the particular cases of $k=n$, and $k=0$, Eq. (2.1) gives:

$$
\begin{aligned}
& A(f) g^{(n)}\left(c_{n}\right)=A(g) f^{(n)}\left(c_{n}\right), \\
& A(f) \Delta_{h}^{n} g\left(c_{0}\right)=A(g) \Delta_{h}^{n} f\left(c_{0}\right) .
\end{aligned}
$$

Proof. We consider the auxiliary function $h:[a, b] \rightarrow \mathbb{R}$,

$$
h=A(f) g-A(g) f \text {. }
$$

It follows that $A(h)=0$. Since $A\left(e_{n}\right) \neq 0$, using (1.1) we deduce that there exist distinct points $\xi_{0}, \ldots, \xi_{n} \in(a, b)$ such that

$$
\left[\xi_{0}, \ldots, \xi_{n} ; h\right]=0 \text {. }
$$

By using Popoviciu's mean value formula (1.3), we deduce that there exists a point $c \in(a, b)$ such that

$$
\Delta_{h}^{n} h(c)=0 .
$$

Next, by using (1.2), we obtain that there exist $c_{k} \in(a, b)$ such that

$$
\Delta_{h}^{n-k} h^{(k)}\left(c_{k}\right)=0, \quad k=1,2, \ldots, n,
$$

and the proof is complete.

Remark 1. Since the functional $A(f):=\left[x_{0}, \ldots, x_{n} ; f\right]$ is obviously of simple form, from Theorem 3, Eq. (2.2), we deduce the Kowalevski result (1.4).

A variant of the Kowalevski result (1.4) in the case of non-differentiable functions, i.e.,

$$
\left[x_{0}, \ldots, x_{n} ; f\right] \Delta_{h}^{n} g\left(c_{0}\right)=\left[x_{0}, \ldots, x_{n} ; g\right] \Delta_{h}^{n} f\left(c_{0}\right),
$$

follows from (2.3). 
We end the paper with an application of Peano's representation formula for continuous linear functionals. For $t \in[a, b]$, let $\varphi_{t}:[a, b] \rightarrow \mathbb{R}$,

$$
\varphi_{t}(x):=(x-t)_{+}^{n-1}:=\left(\frac{x-t+|x-t|}{2}\right)^{n-1}, \quad x \in[a, b] .
$$

If $A: C^{n}[a, b] \rightarrow \mathbb{R}$ is a continuous linear functional with the property that

$$
A\left(e_{i}\right)=0, \quad i=0, \ldots, n-1,
$$

then the following representation formula (see, e.g., $[1,2,5]$ ),

$$
A(f)=\frac{1}{(n-1) !} \int_{0}^{1} A\left(\varphi_{t}\right) f^{(n)}(t) \mathrm{d} t,
$$

holds true.

Remark 2. Let $A: C[a, b] \rightarrow \mathbb{R}$ be a continuous $n$-simple functional and suppose that $f, g \in C^{n}[a, b]$. Then there exist points $c_{k} \in(a, b), k=0, \ldots, n$, such that

$$
\begin{aligned}
& \Delta_{h}^{n-k} g^{(k)}\left(c_{k}\right) \int_{0}^{1} A\left((\cdot-t)_{+}^{n-1}\right) f^{(n)}(t) \mathrm{d} t \\
= & \Delta_{h}^{n-k} f^{(k)}\left(c_{k}\right) \int_{0}^{1} A\left((\cdot-t)_{+}^{n-1}\right) g^{(n)}(t) \mathrm{d} t .
\end{aligned}
$$

Proof. In (2.1) we replace $A$ by its Peano representation (2.4).

\section{ACKNOWLEDGEMENT}

We thank the anonymous reviewers for their constructive comments, which helped us to improve the manuscript.

\section{REFERENCES}

[1] H. Brass and K.-J. Förster, "On the application of the Peano representation of linear functionals in numerical analysis," in Recent progress in inequalities (Niš, 1996), ser. Math. Appl. Dordrecht: Kluwer Acad. Publ., 1998, vol. 430, pp. 175-202.

[2] D. Ferguson, "Sufficient conditions for Peano's kernel to be of one sign," SIAM J. Numer. Anal., vol. 10, pp. 1047-1054, 1973.

[3] G. Kowalewski, Interpolation und genäherte Quadratur. Eine Ergänzung zu den Lehrbüchern der Differential- und Integralrechnung. Leipzig u. Berlin: B. G. Teubner. V, 146 S. u. 10 Abb., 1932.

[4] D. Ş. Marinescu, M. Monea, and C. Mortici, "On some mean value points defined by divided differences and their Hyers-Ulam stability," Results in Mathematics, vol. 70, no. 3-4, pp. 373-384, feb 2016, doi: 10.1007/s00025-016-0532-0. [Online]. Available: http://dx.doi.org/10.1007/s00025-016-0532-0

[5] G. Peano, "Resto nelle formule di quadratura espresso con un integrale definito," Atti della Accademia dei Lincei, Rendiconti (ser. 5), vol. 22, no. 8-9, pp. 562-569, 1913.

[6] T. Popoviciu, Les fonctions convexes, ser. Actualités Sci. Ind., no. 992. Hermann et Cie, Paris, 1944. 
[7] T. Popoviciu, "Notes sur les fonctions convexes d'ordre supérieur. IX. Inégalités linéaires et bilinéaires entre les fonctions convexes. Quelques généralisations d'une inégalité de Tchebycheff," Bull. Math. Soc. Roumaine Sci., vol. 43, pp. 85-141, 1941.

[8] T. Popoviciu, "On the mean-value theorem for continuous functions," Magyar Tud. Akad. Mat. Fiz. Oszt. Közl., vol. 4, pp. 353-356, 1954.

[9] T. Popoviciu, "Remarques sur certaines formules de la moyenne," Arch. Math. (Brno), vol. 5, pp. $147-155,1969$.

[10] D. A. Raikov, "On the local approximation of differentiable functions," C. R. (Doklady) Acad. Sci. URSS (N.S.), vol. 24, pp. 653-656, 1939.

[11] P. K. Sahoo and T. Riedel, Mean value theorems and functional equations. River Edge, NJ: World Scientific Publishing Co. Inc., 1998.

Authors' addresses

Ioan Gavrea

Technical University of Cluj Napoca, Department of Mathematics, Str. Memorandumului nr. 28, 400114 Cluj-Napoca, Romania

E-mail address: ioan.gavrea@math.utcluj.ro

\section{Mircea Ivan}

Technical University of Cluj Napoca, Department of Mathematics, Str. Memorandumului nr. 28, 400114 Cluj-Napoca, Romania

E-mail address: mircea.ivan@math.utcluj.ro

Vicuta Neagos

Technical University of Cluj Napoca, Department of Mathematics, Str. Memorandumului nr. 28, 400114 Cluj-Napoca, Romania

E-mail address: vicuta.neagos@math.utcluj.ro 